\title{
A rollercoaster ride
}

Readers may be interested to hear about one of our patients who has a cautionary tale about rollercoasters/big dippers.

This 37-year-old woman had a Mirena ${ }^{\circledR}$ intrauterine system (IUS) fitted in 2007. The procedure was a straightforward insertion into a $7.5 \mathrm{~cm}$ uterus. She was fully counselled prior to the fitting. 
She was advised to return in 6 weeks' time for a post-insertion check. Unfortunately she did not attend for this check up. In fact she did not attend for a device check until 2010 (26 months following insertion). At this time she was complaining of pelvic pain following a holiday spent riding rollercoasters/big dippers in America.

On examination no threads were seen so an ultrasound scan was requested. The patient had already decided she wanted the IUS removed and had been started on Cerazette ${ }^{\circledast}$ the week before.
No IUS could be seen on the scan; however, an abdominal X-ray showed the device to be well over to the right hand side of the pelvis and likely to be extrauterine in location.

When these findings were discussed with the patient she felt sure that the device had moved during one of her holiday rollercoaster rides, 3 months ago. That was when she had started to feel the pain; she had no problems prior to that. She was referred to gynaecology.

Laparoscopy confirmed the device to be extrauterine and embedded in omentum. It was removed. There has been no recurrence of pain following removal.

Was it the centrifugal force of the rollercoaster ride that made the IUS move?

Josephine Hewson, MRCGP, DFSRH

General Practitioner, Maryport Group Practice, Maryport, UK;

josephine.hewson@gp-A82032.nhs.uk

\section{Competing interests None.}

Provenance and peer review Not commissioned; internally peer reviewed.

J Fam Plann Reprod Health Care 2011;37:255-256 doi:10.1136/fprhc-2011-100170 